\title{
Otoprotective effects of ethosuximide in NOD/LtJ mice with age-related hearing loss
}

\author{
LU SANG $^{1 *}$, TIHUA ZHENG $^{1 *}$, LINGQIAN MIN $^{1 *}$, XIAOLIN ZHANG $^{1}$, XIUFANG MA $^{1,2}$, \\ SHAMI ENTENMAN ${ }^{3}$, YIPENG SU ${ }^{1}$ and QINGYIN ZHENG ${ }^{3}$ \\ ${ }^{1}$ Transformative Otology and Neuroscience Center, Binzhou Medical University, Yantai, Shandong 264000; \\ ${ }^{2}$ Department of Otolaryngology/Head and Neck Surgery, The Affiliated Hospital of Binzhou Medical University, \\ Binzhou, Shandong 256600, P.R. China; ${ }^{3}$ Department of Otolaryngology-HNS, \\ Case Western Reserve University, Cleveland, OH 44106-4952, USA
}

Received December 1, 2015; Accepted May 12, 2017

DOI: $10.3892 / \mathrm{ijmm} .2017 .3004$

\begin{abstract}
Despite long-term efforts to elucidate the mechanisms responsible for age-related hearing loss (AHL), there is currently no available treatment strategy able to provide a cure. Apoptotic cell death, including that of hair cells and spiral ganglion neurons (SGNs) in the cochlea has been proposed to be the classic theory behind the development of AHL. As calcium signaling plays key roles in signal transduction in apoptosis, in this study, we selected ethosuximide, which is able to block T-type calcium ( $\mathrm{Ca}^{2+}$ ion) channels, suppressing $\mathrm{Ca}^{2+}$. We hypothesized that the apoptotic pathway may be blocked through the inhibition of T-type $\mathrm{Ca}^{2+}$ channels in cochlear cells in NOD/LtJ mice. NOD/LtJ mice were divided into 2 groups as follows: the ethosuximide-treated and untreated (control) groups. Ethosuximide was administered by intraperitoneal injection every other day from post-natal day seven (P7) until the mice were 8 weeks of age. Following treatment, auditoryevoked brainstem response (ABR) thresholds and distortion product oto-acoustic emission (DPOAE) of the mice in the
\end{abstract}

Correspondence to: Dr Xiufang Ma, Department of Otolaryngology/Head and Neck Surgery, The Affiliated Hospital of Binzhou Medical University, 661 Huanghe 2nd Road, Binzhou, Shandong 256600, P.R. China

E-mail: drmxf1969@163.com

Professor Qingyin Zheng, Department of Otolaryngology-HNS, Case Western Reserve University, 11100 Euclid Avenue, Cleveland, OH 44106-4952, USA

E-mail: qyz@case.edu

*Contributed equally

Abbreviations: AHL, age-related hearing loss; ABR, auditoryevoked brainstem response; DPOAE, distortion product oto-acoustic emission; OHC, outer hair cell; IHC, inner hair cell; SGN, spiral ganglion neuron

Key words: age-related hearing loss, NOD/LtJ mice, ethosuximide, T-type calcium channels, apoptosis, treatment
2 groups were measured at different time points. Morphometric analysis and the expression of genes involved in the T-type $\mathrm{Ca}^{2+}$-mediated apoptotic pathway were monitored. The ABR and DPOAE results revealed that the NOD/LtJ mice exhibited early-onset and rapidly progressive AHL. A histological examination revealed that hair cell degeneration coincided with the progression of hearing loss. Hair cell and SGN was were significantly lower and auditory function was significantly improved in the ethosuximide-treated group compared to the untreated group. Our data thus indicate that ethosuximide prevents the degeneration of cochlear cells by regulating the expression of genes in apoptotic pathways. Our findings suggest that activating the T-type $\mathrm{Ca}^{2+}$ channel and downstream genes may be key pathological mechanisms responsible for AHL in NOD/LtJ mice.

\section{Introduction}

Presbycusis or age-related hearing loss (AHL) is a common sensory functional disturbance $(1,2)$, which negatively affects the quality of life of affected individuals (3). AHL decreases a person's ability to communicate with a spoken language and has been implicated in various somatopsychic illnesses, including depression, social isolation and a lower self-esteem. Despite extensive research efforts, our understanding of the mechanisms responsible for AHL remains limited, and there are currenlty no available effective medications to prevent or treat this condition. Environmental and other factors, such as endocrine disease and ototoxic drugs may contribute to the development of AHL. In the United States, approximately twothirds of all adults older than 70 years have clinically significant hearing loss (4). Globally, the incidence of AHL has continued to increase due to the aging population, and it has become a major burden to families and society.

Sensorineural hearing loss is characterized by a difficulty in hearing high frequencies and is caused by the degeneration of hair cells and spiral ganglion neurons (SGNs). The organ of Corti is located in the cochlea. It is composed of mechanosensory cells known as hair cells, and is responsible for the sensations of sound. SGNs establish connections between hair cells and central auditory system neurons in the brainstem. 
Unfortunately, hair cells and SGNs are non-renewable; hence, their impairment results in decreased hearing sensitivity $(5,27)$.

The normal lifespan and fecundity of NOD/LtJ mice provide a complementary model to facilitate the genetic basis of human presbycusis. Inbred strains of NOD/LtJ mice are susceptible to early-onset AHL (6). This phenotype has been traced to the hypomorphic $C d h 23$ (753A) allele in the cadherin 23 gene $(C d h 23)$. In addition, the corresponding cadherin $23(\mathrm{CDH} 23)$ is a component of the tip link in haircell stereocilia (7). The major contributor to the difference in hearing loss onset time between NOD/LtJ and C57BL/6J mice is the $A h l 2$ locus on mouse chromosome $(\mathrm{Chr}) 5$ in NOD/LtJ mice (6). NOD/LtJ mice exhibit hearing loss before 3 months, and this is much earlier than C57BL/6J mice, which do not exhibit hearing loss until 10 months of age or older (6). Thus, these features established an appropriate therapeutic time frame for AHL-associated genetic mechanisms, and may be used to provide new insight as to the treatment of AHL.

Calcium channel blockers has been demonstrated to improve hearing thresholds in females during aging, suggesting that calcium disorders are an important factor underlying human AHL (8). Furthermore, $\mathrm{Ca}^{2+}$ ions can activate the apoptotic signaling pathway. Apoptosis plays a key role in the age-related decline of physiological function in multiple organs, including the cochlea (9-11). Thus, in this study, we selected ethosuximide, which is used as a first-line treatment for the absence seizures, and which its primary mechanism is to selectively inhibit T-type $\mathrm{Ca}^{2+}$ channels in the neuronal cell membrane (12). This study aimed to evaluate the otoprotective effects of ethosuximide as a $\mathrm{Ca}^{2+}$ channel blocker in NOD/LtJ mice with AHL.

The pan-caspase inhibitor, Z-Val-Ala-Asp (OMe)fluoromethyl ketone (Z-VAD-FMK), can attenuate the progression of AHL in $C d h 23^{\text {erl }} /$ rl mice and reduce age-related outer hair cell (OHC) degeneration, and prevent apoptotic cell death by inhibiting the calpain signaling pathway (13). Calpain is a cysteine protease, and alterations in intracellular $\mathrm{Ca}^{2+}$ can activate calpain and the caspase family $(14,15)$. In addition, erythropoietin (EPO) can protect against hearing loss in $C d h 23^{e r l} /$ rl mice through apoptosis-related pathways (16).

In the present study, NOD/LtJ mice were treated with ethosuximide from post-natal day 7 (P7). We hypothesized that ethosuximide could block T-type $\mathrm{Ca}^{2+}$ channels, suppress apoptotic factors, and thus prevent hair cell degeneration and preserve auditory function.

\section{Materials and methods}

Animals. NOD/LtJ mice (age range, P7 to 5 months) obtained from the Jackson Laboratory (Bar Harbor, ME, USA), were housed at $24^{\circ} \mathrm{C}$ with a 12 -h light/dark cycle in a standard animal house. A total of $95 \mathrm{NOD} / \mathrm{LtJ}$ mice were used in this study. Food and water were available ad libitum. The Animal Care and Use Committee of Binzhou Medical University, Yantai, China approved the care and use of mice for this study.

Allocation of mice for use in experiments. In the experiment shown in Fig. 1, we tested ABR and DPOAE at 3, 4, 6, 8, 10 and 14 weeks of age for the NOD/Shi LtJ mice ( $n=5 /$ time point) and the mice were anesthetized and sacrificed for phalloidin staining after testing. In order to determine the tendency for cell loss, the mice were anesthetized at 18 weeks $(n=5)$ and sacrificed for phalloidin staining after testing. The key time points are shown. As mouse hearing can be tested at 3 weeks of age by ABR and we did not observe any loss of hair cells, this was not shown (total mice used $=35$ ). In the experiment shown in Fig. 2, the ABR and DPOAE of the untreated mice and ethosuximide-treated mice were assessed at 4, 6 and 8 weeks of age ( $n=5 /$ group). After testing at 4 weeks, the mice were then allowed to grow 6 weeks for testing and then to 8 weeks for testing (total mice used $=10$ ). In the experiment shown in Fig. 3, the mice in the ethosuximide-treated and untreated groups were sacrificed at 4 and 8 weeks of age $(n=5 / g r o u p /$ time point), and the Corti were isolated, $\mathrm{OHC}$ and $\mathrm{IHC}$ hair bundles were visualized with phalloidin staining (total mice used $=20$, 10 at each time point in both groups). In the experiment shown in Fig. 4, at 4 and 8 weeks of age, the mice in the ethosuximidetreated and untreated groups were sacrificed ( $n=5 /$ group/time point) (total mice used $=20,10$ at each time point in both groups). In the experiment shown in Fig. 5, we examined expression differences between the ethosuximide-treated and untreated mice ( $n=5 /$ group) at the age of 8 weeks (total mice used $=10)$.

Assessment of auditory function. Auditory-evoked brainstem response (ABR) was tested to identify the lowest level at which an ABR pattern can be recognized. Distortion product otoacoustic emission (DPOAE) represented the function of OHCs. They were measured at various intervals at 3, 4, 6, 8, 10 and 14 weeks of age for the NOD/Shi LtJ mice ( $n=5 /$ time point). The computer-aided evoked potential system (Intelligent Hearing Systems, Miami, FL, USA) was used to test for ABR thresholds $(17,18)$. Briefly, the mice were anesthetized with $3 \%$ chloral hydrate $(10 \mathrm{mg} / \mathrm{kg})$ and body temperature was maintained at $37 \pm 1^{\circ} \mathrm{C}$. Platinum needle electrodes were inserted subcutaneously at the vertex (active), and ventrolateral to the right ear (reference) and left ear (ground). ABR thresholds were detected by reducing the sound pressure level (SPL) at $10-\mathrm{dB}$ steps, and subsequently at 5-dB steps. Following the ABR test, we measured DPOAE using the IHS Smart EP 3.30 and USB ez Software (Intelligent Hearing Systems, Miami, FL, USA) (19). Stimuli (ranging from 4,422 to $35,344 \mathrm{~Hz}$ ) were presented from lowest to highest frequency. Amplitudes were recorded automatically.

Morphometric analysis. For morphometric observation, at 4, $8,10,14$ and 18 weeks, the mice were anesthetized and sacrificed ( $n=5 /$ time-point). The inner ears were removed, fixed in $4 \%$ paraformaldehyde, and decalcified in Cal-Ex solution. Cochleae were isolated and divided into 3 turns (apex, middle turn and base turn). Finally, the organ of Corti was exposed in each turn and specimens were mounted on glass slides. $\mathrm{OHC}$ and inner hair cell (IHC) hair bundles were visualized with Alexa Fluor ${ }^{\circledR}$ 488-conjugated phalloidin staining and a confocal laser scanning microscope (Leica TCS SPE; Leica Microsystems GmbH, Wetzlar Germany). The number of missing hair cells was counted per view using a x40 oil immersion objective lens (13); counts of continuous views for each turn were recorded for statistical analyses. Cytocochleograms were generated. 
Table I.Primers used for RT-qPCR for the detection of apoptosisrelated genes.

\begin{tabular}{llc}
\hline \multicolumn{1}{c}{ Primer sequences } & $\begin{array}{c}\text { Product } \\
\text { size (bp) }\end{array}$ \\
ID & \multicolumn{1}{c}{132} \\
GAPDH-F & 5'-CTTCCGTGTTCCTACCCCCAATGT-3' & 132 \\
GAPDH-R & 5'-GCCTGCTTCACCACCTTCTTGATG-3' & \\
$\alpha 1 \mathrm{G}-\mathrm{F}$ & 5'-GGGCTGTATTCCCCTCCATCGT-3' & 97 \\
$\alpha 1 \mathrm{G}-\mathrm{R}$ & 5'-CTTCTGACCCATTCCCACCATCAC-3' & \\
$\alpha 1 \mathrm{H}-\mathrm{F}$ & 5'-GCTATGTTGCCCTGGATTTTGAGC-3' & 130 \\
$\alpha 1 \mathrm{H}-\mathrm{R}$ & 5'-GGAAGGAAGGCTGGAAGAGTGC-3' & \\
$\alpha 1 \mathrm{I}-\mathrm{F}$ & 5'-CAAGTTCTCTTTGGTTGGCATCG-3' & 123 \\
$\alpha 1 \mathrm{I}-\mathrm{R}$ & 5'-GGGTAAGTGTTGCGTTCCCTTCAT-3' & \\
m-calpain-F & 5'-GTTTGTGACCGCCAAGAAAAATGG-3' & 107 \\
m-calpain-R & 5'-GAAGAGTTCCGAGTCCCCTGCTGT-3' & \\
$\mu$-calpain-F & 5'-TCCTGGTCAATACCCTCAGC-3' & 95 \\
$\mu$-calpain-R & 5'-AGGCTGGTGAAGACGATGTT-3' & \\
Caspase-3-F & 5'-TGAATCCACTGAGGTTTTGTTG-3' & 93 \\
Caspase-3-R & 5'-TGCTGGTGGGATCAAAGC-3' & \\
Caspase-9-F & 5'-CATATCTGCATGTCCCCTGA-3' & 104 \\
Caspase-9-R & 5'-AGCCAGAGGTTCTCAGACCA-3' & \\
Caspase-12-F 5'-AAAAATCCTGGGATCTTGGA-3' & 97 \\
Caspase-12-R 5'-GGGAATTAGCACAGGCAACT-3' & \\
\hline
\end{tabular}

GAPDH, glyceraldehyde 3-phosphate dehydrogenase.

Drug application. Seven-day-old mice were divided into 2 groups as follows: ethosuximide-treated group and untreated group ( $\mathrm{n}=5$ /group). Ethosuximide (Sigma Chemical Co. St. Louis, MO, USA) was dissolved in $\mathrm{ddH}_{2} \mathrm{O}$ and stored at $4^{\circ} \mathrm{C}$ in dark bottles until use. Ethosuximide was administered by intraperitoneal injection $(10 \mathrm{mg} / \mathrm{kg}$ of body weight) every other day from P7 to 8 weeks of age. A higher dosage $14 \mathrm{mg} / \mathrm{kg}$ injection of ethosuximide every other day resulted in the death of 2 litters of mice (not the mice used in these experiments) after 2 weeks initial treatment. We measured body weight prior to injection. Body weight was also used for monitoring the animal health status.

Evaluation of the protection of auditory function. The ABR and DPOAE of the untreated mice and ethosuximidetreated mice were assessed at 4,6 and 8 weeks of age ( $\mathrm{n}=5 /$ group/time point).

The mice in the ethosuximide-treated and untreated groups were sacrificed at 4 and 8 weeks of age ( $\mathrm{n}=5 /$ group/time point), and the Corti were isolated, $\mathrm{OHC}$ and IHC hair bundles were visualized with phalloidin staining as described previously. Hair cells in the ethosuximide-treated and untreated groups were observed and counted, and these were reported as the percentage of missing OHCs and IHCs.

At 4 and 8 weeks of age, the mice in the ethosuximide-treated and untreated groups were sacrificed ( $\mathrm{n}=5 /$ group/time point) and the bullae (including both the middle and inner ears) were isolated, and immersed in $4 \%$ paraformaldehyde, decalcified with Cal-Ex solution, and embedded in paraffin. Subsequently, 5 -mm-thick sections of cochleae were cut, mounted on glass slides, counterstained with hematoxylin and eosin (H\&E), and observed under a microscope using a 40X objective lens and 100X oil immersion objective lens (Leica DM4000 B; Leica Microsystems $\mathrm{GmbH}$ ).

Reverse transcription-quantitative PCR (RT-qPCR). The experiment were assessed between the ethosuximide-treated and untreated mice ( $\mathrm{n}=5 /$ group) at the age of 8 weeks. The entire inner ears were dissected, and total RNA was extracted using TRIzol reagent (Invitrogen, Carlsbad, CA, USA). For each sample, a reverse transcriptase reaction was performed with $1 \mu \mathrm{g}$ of total RNA using the ReverTra Ace qPCR RT kit (Toyobo, Osaka, Japan). The resulting cDNA was diluted for use as template for the real-time PCR reaction. Primer3 was used to design the primers (http://primer3.sourceforge. net/) (Table I). The PCR reaction was carried out using the FastStart Universal SYBR-Green Master kit (Roche, Mannheim, Germany) and specific primers in a Bio-Rad iCycler iQ5 peltier thermal cycler. The process was as follows: one cycle at $95^{\circ} \mathrm{C}$ for $10 \mathrm{~min} ; 40$ cycles at $95^{\circ} \mathrm{C}$ for $15 \mathrm{sec}, 58^{\circ} \mathrm{C}$ for $30 \mathrm{sec}$ and $72^{\circ} \mathrm{C}$ for $10 \mathrm{sec}$; and 71 cycles at $60-95^{\circ} \mathrm{C}$. PCR cycles were continued until the fluorescence intensity exceeded a predetermined threshold, and he cycle number was measured automatically. The relative quantification of the initial amount of template for each sample was achieved by determining the number of cycles. The $\Delta \mathrm{Ct}$ value was calculated using the comparative $\mathrm{Ct}$ threshold method. The difference in the initial amount of total RNA among samples was normalized using the housekeeping gene, glyceraldehyde 3-phosphate dehydrogenase (GAPDH). The relative levels of target gene mRNA were analyzed using the $2-\Delta \Delta \mathrm{Ct}$ method (20).

Statistical analysis. Statistical comparisons of differences between groups were conducted using the Student's t-test. A P-value $<0.05$ was considered to indicate a statistically significant difference. All statistical analyses were performed using the statistical package SPSS for Windows (version 16.0; SPSS, Inc., Chicago, IL, USA).

\section{Results}

NOD/LtJ mice are characterized by increased hearing impairment with age. To investigate hearing loss during the lifespan of NOD/LtJ mice, we detected ABR and DPOAE at 6 time points (in mice aged between 3 and 14 weeks, $n=5 /$ group/time point) (Fig. 1A). The 3-week-old mice exhibited hearing loss with average ABR thresholds $>60 \mathrm{~dB}$ above normal, which is considered to be a profound impairment (6). The ABR thresholds increased after 8 weeks of age, particularly at high frequencies (16 and $32 \mathrm{kHz}$ ). From 3 to 8 weeks of age, the average shift in the ABR threshold was approximately $15 \mathrm{~dB}$; while from 8 to 14 weeks of age, the average shift in the ABR threshold was $>25 \mathrm{~dB}$ (Fig. 1A). In the 12-week-old mice, the average ABR thresholds were $>90 \mathrm{~dB}$ SPL, and the 14-week-old mice exhibited almost complete deafness. DPOAE was measured to determine $\mathrm{OHC}$ function over time (Fig. 1B). The 3-week-old mice exhibited decreased DPOAE amplitudes, which deteriorated with age.

NOD/LtJ mice are characterized by the abnormal morphology of the cochleae. To investigate changes in cochleae morphology 

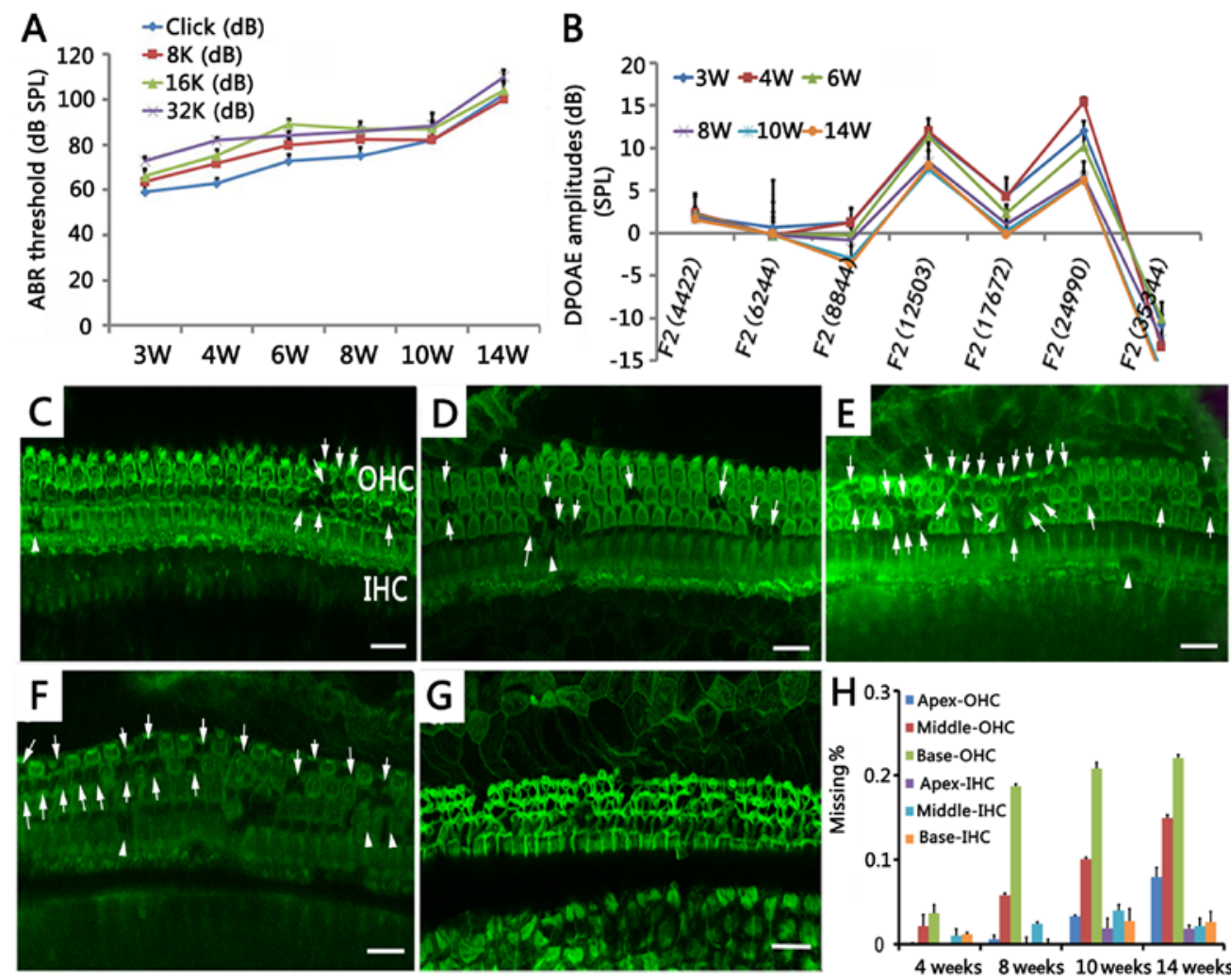

Figure 1. Auditory function tests in NOD/LtJ mice at different time points. (A) Mean auditory-evoked brainstem response (ABR) thresholds for mice aged between 3 and 14 weeks, exhibiting early hearing loss (at 3 weeks of age) progressing to severe hearing loss by 14 weeks of age. (B) Distortion product otoacoustic emission (DPOAE) amplitudes decreased in mice aged between 3 and 14 weeks. W, week. (C-H) Hair cells in the basal turn of the organ of Corti in 4-, 8-, 10-, 14- and 18-week-old mice were stained to reveal stereocilia bundles. At 18 weeks of age, normal hair cells in the basal turn had almost disappeared. Dark areas indicate outer hair cell (OHC) degeneration. Arrows indicate sites of OHC loss, and triangles indicate sites of inner hair cell (IHC) loss (scale bar, $20 \mu \mathrm{m}$ ). (H) Cytocochleograms showing the mean percentage of total hair cell loss (y-axis) at various time-points (x-axis) in the basal turn. Error bars indicate the standard error of the mean.

during the course of hearing loss to the status of complete deafness in NOD/LtJ mice, the organ of Corti was examined in all 3 turns of the cochleae (Fig. 1C-G). To quantify hair cell loss, cytocochleograms were generated from mice aged between 4 and 14 weeks (Fig. 1H). From post-natal day zero (P0) to 3 weeks of age, the mice exhibited no evidence of OHC loss. However, in the 4-week-old mice, evidence of OHC degeneration began at the basal turn and $\mathrm{OHC}$ loss was $6 \%$ in all 3 turns totally (Fig. 1C and $\mathrm{H}$ ). At 8 and 14 weeks of age, OHC loss was 25 and 45\%, respectively, in all 3 turns (Fig. 1D and $\mathrm{H}$ ). The loss of OHCs coincided with increased ABR thresholds. In the 18-week-old mice, no normal OHCs were visible (Fig. 1G). In the 10-week-old mice, evidence of IHC degeneration was observed (Fig. 1H). IHC loss was $9 \%$ in the 10 -week-old mice, which increased with age.

Ethosuximide treatment protects against decreased auditory function in NOD/LtJ mice. To investigate whether ethosuximide protects against hearing loss in NOD/LtJ mice, we conducted hearing tests in 4-, 6- and 8-week-old ethosuximidetreated and untreated mice ( $\mathrm{n}=5 /$ group).

ABR thresholds were lower in the ethosuximide-treated group than in the untreated group at stimulus frequencies of $8 \mathrm{kHz}$ in the 4 -week-old mice $(\mathrm{P}<0.01), 16 \mathrm{kHz}$ in the 6 -week-old mice $(\mathrm{P}<0.01)$, and at click, 8 and $32 \mathrm{kHz}$ in the 8 -week-old mice $(\mathrm{P}<0.05)$ (Fig. $2 \mathrm{~A}-\mathrm{D})$. ABR threshold shifts in the 2 groups during the period (4-8 weeks) of drug application were also measured to determine whether ethosuximide can alter or attenuate the progression of hearing loss. The ethosuximide-treated group displayed lower ABR threshold shifts compared to the untreated group (Fig. 2E).

The mean amplitude of DPOAE was significantly higher in the ethosuximide-treated group than in the untreated group at 8,844 and $12,503 \mathrm{~Hz}$ in the 4-week-old mice $(\mathrm{P}<0.01)$, at 4,422, 17,672 and $24,990 \mathrm{~Hz}$ in the 6-week-old mice $(\mathrm{P}<0.05)$, and at $8,844,35,344 \mathrm{~Hz}(\mathrm{P}<0.01)$ and $12,503 \mathrm{~Hz}(\mathrm{P}<0.05)$ in the 8 -week-old mice (Fig. 2G-I). As DPOAE reflects OHC function, these data suggest that ethosuximide protects auditory function in NOD/LtJ mice.

Fluorescence images of the OHCs and IHCs characterized the progression of hair cell degeneration in the cochleae in the 4- and 8-week-old untreated mice (Fig. 3A and C). OHC survival was increased in the ethosuximide-treated group compared to the untreated group (Fig. 3B and D). Cytocochleograms indicated that the mean percentage of $\mathrm{OHC}$ loss was significantly lower in the ethosuximide-treated group than in the untreated group at all 3 cochlear turns. IHC loss was not substantially altered by ethosuximide treatment (Fig. 3E and F) (n=5/group/ time point).

H\&E staining demonstrated that the ethosuximide-treated group (Fig. 4B and D) experienced minor SGN and hair cell degeneration in the basal turn compared to the untreated 

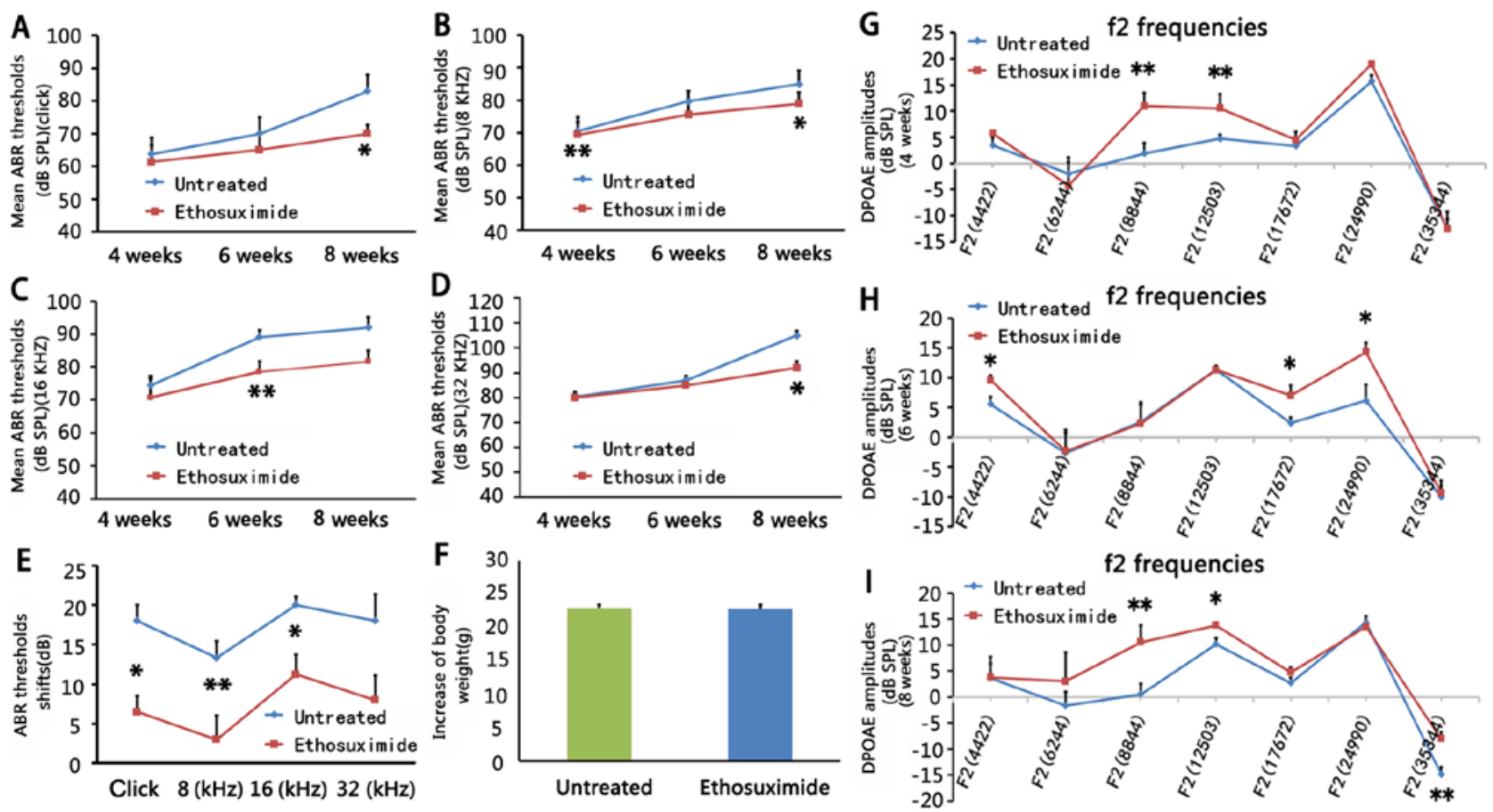

Figure 2. Effects of ethosuximide treatment in different frequencies at different time points (n=5/group). (A-E and G-I) In 4-week-old mice, auditory-evoked brainstem response $(\mathrm{ABR})$ thresholds in the ethosuximide-treated group (red line) were significantly lower compared to the untreated group (blue line) at a stimulus frequency of $8 \mathrm{kHz}(\mathrm{P}<0.01)$. In 6-week-old mice, $\mathrm{ABR}$ thresholds in the ethosuximide-treated group (red line) were significantly lower compared to the untreated group (blue line) at a stimulus frequency of $16 \mathrm{kHz}(\mathrm{P}<0.01)$. In 8-week-old mice, ABR thresholds in the ethosuximide-treated group (red 2 groups during the period (4-8 weeks) of drug application. (F) There were no significant differences in body weight (8-week-old) in ethosuximide-treated mice compared to untreated mice after drug application. Comparison of distortion product oto-acoustic emission (DPOAE) amplitudes between the 2 mouse groups in 4-week-old mice (G), 6-week-old mice (H) and 8-week-old (I) mice. (G) In 4-week-old mice, mean DPOAE amplitudes in the ethosuximide-treated group (red line) at 8,844 and $12,503 \mathrm{~Hz}$ were significantly higher compared to the untreated group (blue line). (H) In 6-week-old mice, mean DPOAE amplitudes in the ethosuximide-treated group (red line) at 4,422, 17,672 and 24,990 Hz were significantly higher compared to the untreated group (blue line). (I) In 8-week-old mice, mean DPOAE amplitudes in the ethosuximide-treated group (red line) at 8,844,12,503 and 35,344 $\mathrm{Hz}$ were significantly higher compared to the untreated group (blue line). Error bars indicate the standard error of the mean. ${ }^{*} \mathrm{P}<0.05$ and ${ }^{* *} \mathrm{P}<0.01$.
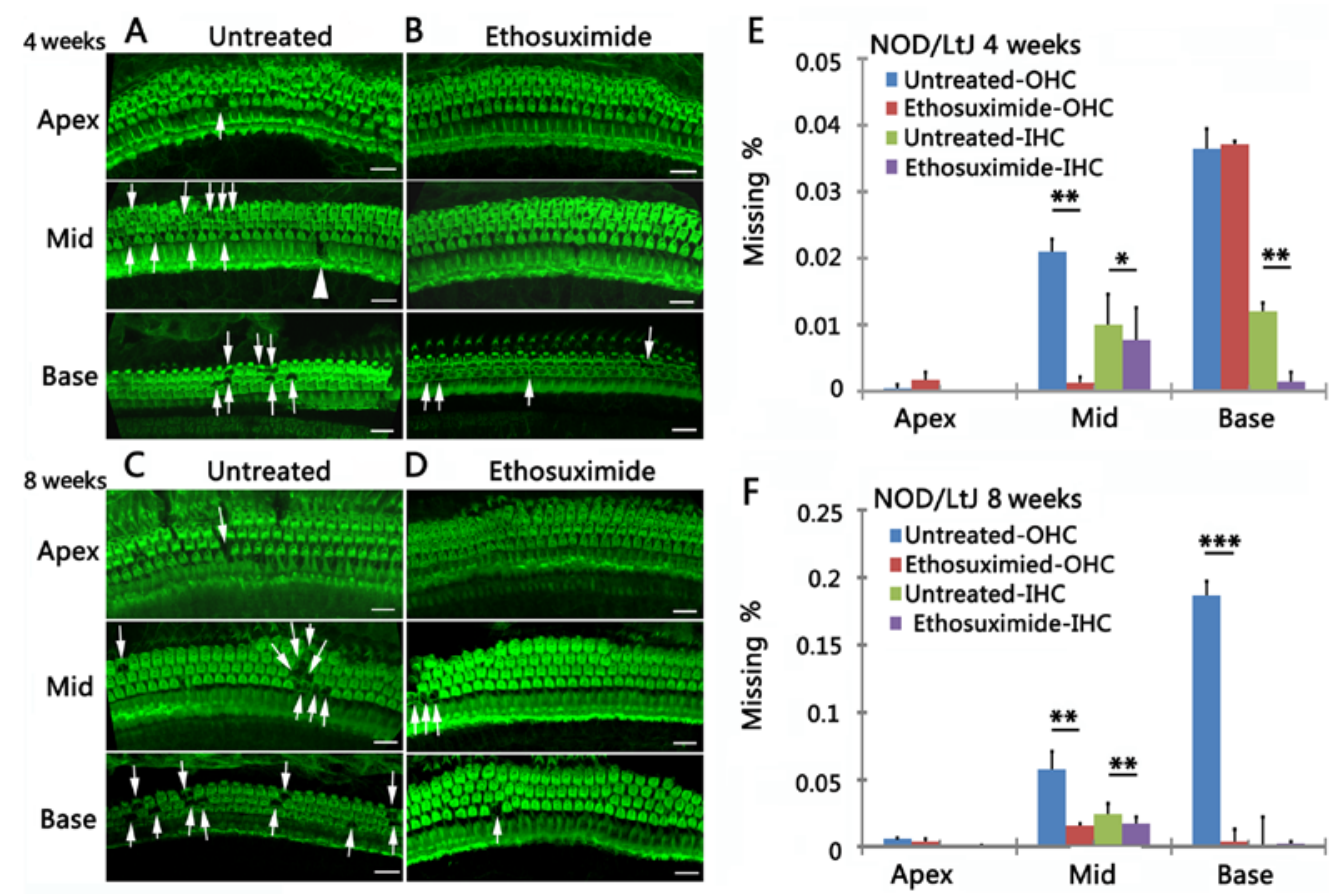

Figure 3. Morphological observations of effects of ethosuximide treatment compared to the untreated group. (A-B and C-D) In untreated mice, outer hair cell (OHC) morphology and arrangement appeared abnormal (A and C) compared to ethosuximide-treated mice (B and D). Arrows indicate sites of OHC loss, and triangles indicate sites of inner hair cell (IHC) loss (scale bar, $20 \mu \mathrm{m}$ ). (E and F) OHCs and IHCs in the apical-basal cochlear location in 4- and 8-week-old mice are shown. Mean OHC loss (\%) in the middle and basal turns were significantly lower in ethosuximide-treated mice compared to untreated mice. Error bars indicate the standard error of the mean. ${ }^{*} \mathrm{P}<0.05,{ }^{* *} \mathrm{P}<0.01$ and ${ }^{* * *} \mathrm{P}<0.001$. 
A

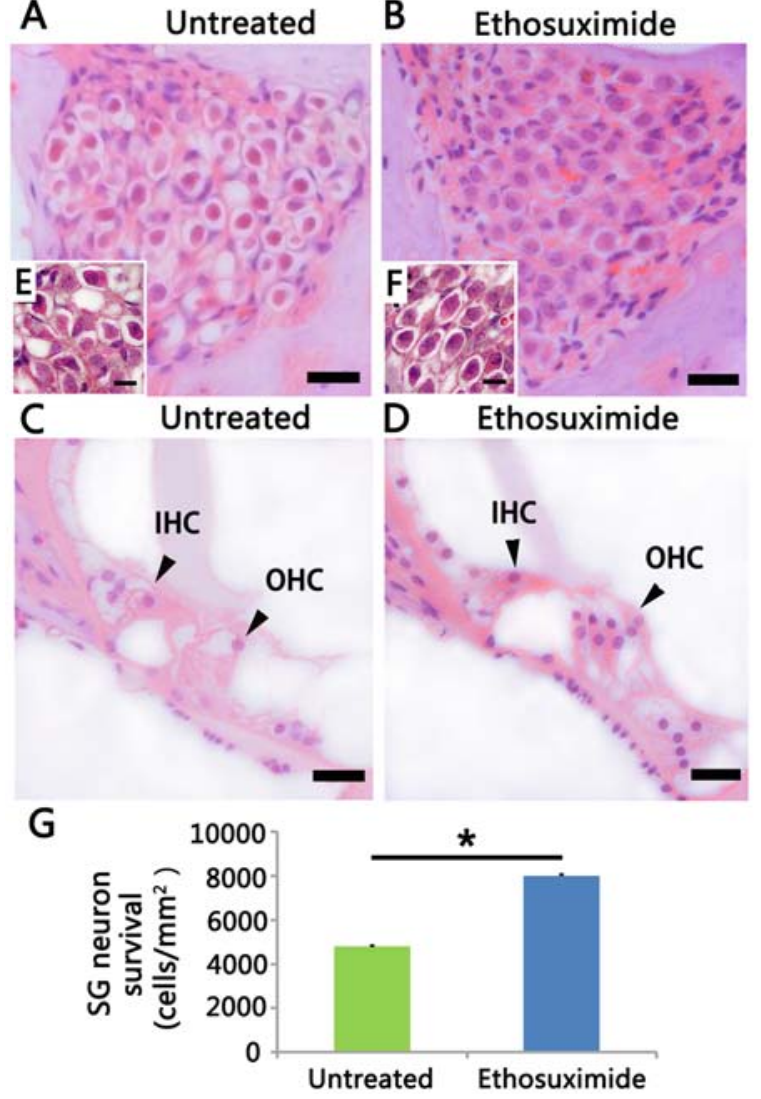

Figure 4. Ethosuximide treatment reduces cochlear cell degeneration. Spiral ganglion neurons (SGNs) (A and B) and hair cells (C and D) in the basal turn of the cochleae from ethosuximide-treated and untreated NOD/LtJ mice. Arrows indicate regions of hair cells (scale bar, $25 \mu \mathrm{m}$ ). (A and C) Photomicrograph of the basal turn of the cochleae in 8-week-old mice in the untreated group shows degeneration of SGNs and hair cells. (B and D) Photomicrograph of the basal turn of the cochleae in 8-week-old mice in the ethosuximide-treated group shows less degeneration of SGNs and hair cells. (E and F) Photomicrograph of the SGN of ethosuximide-treated and untreated mice demonstrates apoptotic features in the untreated group at a $100 \mathrm{X}$ oil immersion objective lens; scale bar, $1 \mathrm{~mm}$ ). (E) In the untreated group, SGNs revealed apoptotic features: shrunken cytoplasm, condensed chromatin and pyknosis, and decreased neuronal number compared to the ethosuximide-treated group (F). (G) In 8 -week-old mice in the ethosuximide-treated group, SGN survival was increased compared to the untreated group. Error bars indicate the standard error of the mean. ${ }^{*} \mathrm{P}<0.05$.

group (Fig. 4A and C) in the 8-week-old mice (n=5/group/ time point). In the untreated group, the SGNs did not exhibit apoptotic features in the basal turn until the mice were 8 weeks old (Fig. 4A). At this time poinr, auditory nerve fibers became loosened and the neurons exhibited a shrunken cytoplasm, condensed chromatin and pyknosis (Fig. 4A and E). In the basal turn, the neuronal population was decreased in the untreated group compared to the ethosuximide-treated group (Fig. 4G). However, the SGN in the apical turn was normal at this time point. Cell counting demonstrated that ethosuximide treatment increased SGN survival (Fig. 4G) in the 8-week-old mice, and increased OHC survival in both the 4- and 8-weekold mice (Fig. 3E and F).

Ethosuximide treatment was not associated with any noticeable side-effects in the 4- and 8-week-old mice. There were no significant differences in body weight in the ethosuximidetreated mice compared to the untreated mice (Fig. 2F).
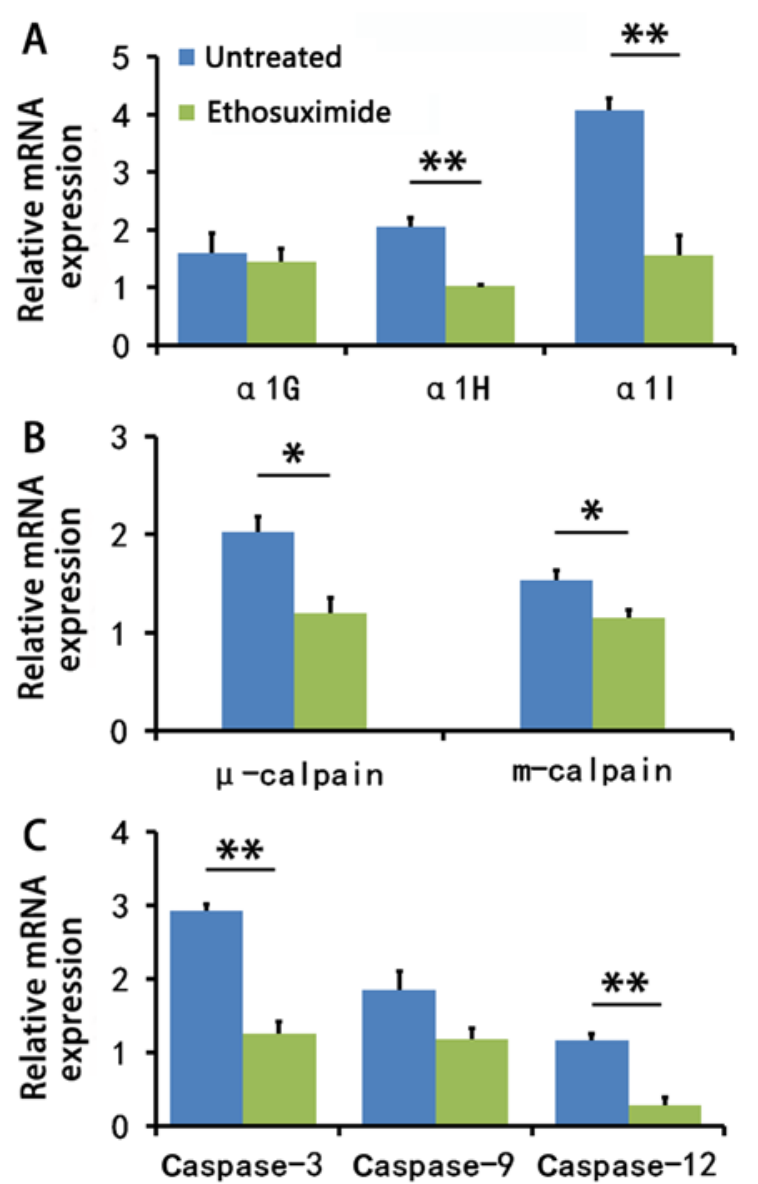

Figure 5. T-type calcium-mediated apoptotic pathway was downregulated in ethosuximide-treated 8-week-old NOD/LtJ mice. (A) Mean expression levels of $\alpha 1 \mathrm{G}, \alpha 1 \mathrm{H}$ and $\alpha 1 \mathrm{I}$ subunits were determined by RT-qPCR. There was a significant decrease in $\alpha 1 \mathrm{H}$ and $\alpha 1 \mathrm{I}$ gene expression in the ethosuximidetreated group compared to the untreated group. (B) Mean mRNA levels of $\mu$-calpain and m-calpain were significantly decreased in the ethosuximidetreated group, indicating that the intrinsic apoptotic pathways was involved in hearing loss in NOD/LtJ mice. (C) Mean mRNA levels of caspase-3, -9 and -12 in inner ear tissues of the ethosuximide-treated group were lower compared to the untreated group. Error bars indicate the standard error of the mean. ${ }^{*} \mathrm{P}<0.05$ and ${ }^{* *} \mathrm{P}<0.01$.

Ethosuximide treatment decreases calpain-mediated apoptosis-related gene expression. We then determined whether the blocking of T-type $\mathrm{Ca}^{2+}$ channels can downregulate cochlear cell gene expression. The family of T-type $\mathrm{Ca}^{2+}$ channels is comprised of 3 main pore-forming $\alpha$ subunits: $\alpha 1 \mathrm{G}$, $\alpha 1 \mathrm{H}$ and $\alpha 1 \mathrm{I}$ (21). The mean mRNA expression levels of the $\alpha 1 \mathrm{G}, \alpha 1 \mathrm{H}$ and $\alpha 1 \mathrm{I}$ subunits were decreased in the cochleae of the ethosuximide-treated 8-week-old NOD/LtJ mice; a significant difference was observed between the ethosuximidetreated group and the untreated group for the $\alpha 1 \mathrm{H}$ and $\alpha 1 \mathrm{I}$ genes $(\mathrm{P}<0.01)$ (Fig. 5A).

There are 2 major intracellular apoptotic pathways. Extracellular stimulican initiate an extrinsic pathway (caspase-8) that activates the caspase family. Alternatively, the intrinsic pathway involves intracellular calpain, which ultimately triggers apoptosis by activating caspase-3 (Fig. 6) $(22,23)$. Calpains (m-calpain and $\mu$-calpain) are activated by $\mathrm{Ca}^{2+}(24)$. Our results suggested that ethosuximide treatment downregulated the intrinsic apoptotic pathway (Fig. 5B and C). 


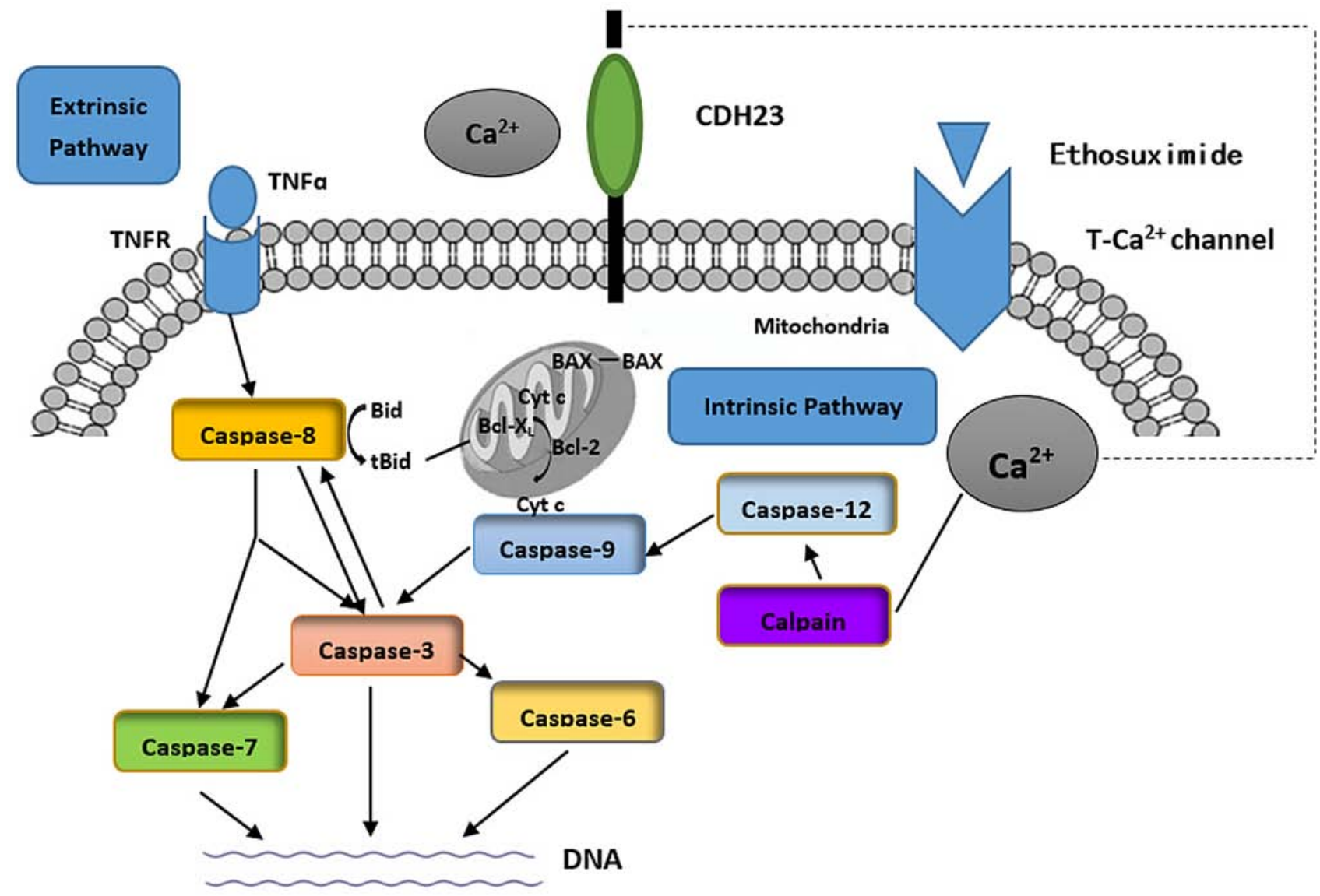

Figure 6. Conceptual graphic of targeted sites of ethosuximide inhibition and associated molecules mechanism in NOD/LtJ mice. Calpains are activated by $\mathrm{Ca}^{2+}$, which subsequently activates caspase-12, -9 and -3 ; and finally initiates cell death. Ethosuximide treatment blocks this $\mathrm{T}_{\text {-type }} \mathrm{Ca}^{2+}$ channel, and associated genes are downregulated. Ethosuximide treatment has no effect on extrinsic caspase-8. Dashed lines are proposed links indicating that that $\mathrm{Ca}^{2+}$ may influence the efficiency of $\mathrm{CDH} 23$.

\section{Discussion}

This study included NOD/LtJ mice with a Cdh23 and Ahl2 background, and this strain experienced progressive hearing loss starting at approximately 3 weeks after birth. AHL began in the high frequency region and progressed to the low frequency region with increasing age. The phenotype is similar to that observed in some humans with presbycusis, in which the basal turn of the cochlea first degenerates (25). These functional deficits were accompanied by the loss of hair cells and SGNs that began at the base of the cochlea, which spread toward the apex. NOD/LtJ mice were deaf by 14 weeks of age.

AHL is characterized by the widespread structural and functional degeneration of hair cells and SGNs in the cochleae (26). Hair cells are responsible for collecting acoustic signals and transducing them to SGNs. SGNs transform acoustic signals into nerve impulses that are received by the auditory cortex. Finally, information is interpreted in the brain (27). Hair cells promote neurotrophin release; and 93\% of SGNs innervate IHCs (28), which suggests that the degeneration of SGNs may be a secondary phenomenon associated with the loss of IHCs. However, in NOD/LtJ mice, SGN degeneration occurred earlier than the loss of IHCs, suggesting the occurrence of early-stage SGN degeneration, which is independent of the age-related loss of hair cells.
As NOD/LtJ mice exhibit rapid and progressive hearing loss, associated genetic mechanisms may provide new insight into the development of AHL and may aid in prevention and treatment. AHL is a genetically complex quantitative trait in common inbred mouse strains, including NOD/LtJ mice. Based on our observations, auditory functional deficiencies occurred before cochlear cell degeneration in the NOD/LtJ mice. We hypothesize that synergistic interactions of $C d h 23$ and $A h l 2$ variants are responsible for the AHL phenotype. Interactions between $C d h 23$ and $A h l 2$ have not been characterized, as $A h l 2$ has not been identified at the gene level. In NOD/LtJ mice, exon 7 in $C d h 23$ has a single nucleotide polymorphism that displays an important association with AHL. AHL may be caused by homozygosity in $C d h 23$ (753A) in association with the effects of heterogeneous secondary factors (7). Cadherins mediate $\mathrm{Ca}^{2+}$-dependent cell-cell adhesion, migration and compaction (29). CDH23 is encoded by the $C d h 23$ gene, which is a transmembrane protein and a component of the tip-link in hair cells in the organ of Corti (29-31). CDH23 is involved in regulating the activity of mechanically gated ion channels in hair cells $(6,32,33)$. Evidence suggests that $\mathrm{Ca}^{2+}$-dependent cell-cell adhesion deficiency significantly influences cochlear cell integrity (34).

Population-based studies have linked the use of $\mathrm{Ca}^{2+}$ channel blockers to improved hearing thresholds in elderly females. 
T-type $\mathrm{Ca}^{2+}$ channels are voltage-gated $\mathrm{Ca}^{2+}$ channels (VGCCs) that can regulate intracellular $\mathrm{Ca}^{2+}$ levels (35-38). In this study, we demonstrated that a blocker of T-type $\mathrm{Ca}^{2+}$ channels, ethosuximide, significantly reduced the increase in age-related $\mathrm{ABR}$ thresholds, as well as OHC and SGN loss, in NOD/LtJ mice; this most likely mediated through the effects of the downstream apoptotic pathway. Ethosuximide is a relatively selective inhibitor of T-type $\mathrm{Ca}^{2+}$ channels. In accordance with our findings, it has been demonstrated that the blocking of T-type $\mathrm{Ca}^{2+}$ channels with ethosuximide can prevent epileptogenesis (39). In a study on glutamate-induced injury in stroke, the blocking of $\mathrm{Ca}^{2+}$ channels effectively prevented $\mathrm{Ca}^{2+}$ entry and delayed neuronal death (40). In addition, as shown in another study, the protective effect of hydrogen sulfide on colonic mucosa relied on T-type $\mathrm{Ca}^{2+}$ channel-dependent neuronal excitation in rats (41). In a noise-induced C57BL/6J mouse model, ethosuximide delayed age-related SGN loss and preserved ABR thresholds (42). However, $\mathrm{Ca}^{2+}$ was not the sole mediator in ferutinin-mediated eryptosis/erythroptosis, as the wastage of external $\mathrm{Ca}^{2+} \mathrm{did}$ not prevent the apoptotic effect of ferutinin (43).

The mechanisms responsible for AHL in NOD/LtJ mice include structural alterations of the tip-links in hair cells and cochlear cell apoptosis. $\mathrm{CDH} 23$ protein is located near the $\mathrm{Ca}^{2+}$ binding site. We speculate that $\mathrm{Ca}^{2+}$ may influence the efficiency of $\mathrm{CDH} 23$ interactions with proteins, affect $\mathrm{Ca}^{2+}$-dependent cell adhesions, and ultimately lead to apoptosis in NOD/LtJ mice. Thus we suggest that blocking the $\mathrm{Ca}^{2+}$ channel with ethosuximide may inhibit the calpain-mediated (intrinsic) apoptotic pathway that activates the caspase family and a cascade that leads to cell death. This is supported by data indicating that leupeptin, which is a calpain inhibitor, protects the cochlear and vestibular hair cells against gentamicin (GM) ototoxicity (44). Furthermore, conventional $\mathrm{Ca}^{2+}$ entry antagonists prevent neuronal death when administered before and during the injury phase of glutamate excitotoxicity $(45,46)$. However, following excitotoxic insults, these $\mathrm{Ca}^{2+}$ entry antagonists are no longer effective. Thus, our results demonstrate that the prophylactic use of ethosuximide may prevent neuronal death.

The debate of determining which component of the cochlea is involved in the aging process and which part of the inner ear is involved if affected by ethosuximide continues. It should be noted that the elevation of hearing thresholds in AHL detected by traditional audiograms is primarily based on OHC and not SGNs. Further studies are required in order to further understand the subtle structural changes in AHL and also following the application of ethosuximide.

In conclusion, the NOD/LtJ mouse provides a valuable model for the study of AHL-associated genetic mechanisms. In particular, the role of cadherins in the tip-link of hair cells can be investigated. The effects of mutation in $A h l 2$ remain unclear, and warrant further investigation. This study confirmed that blocking T-type $\mathrm{Ca}^{2+}$ channels plays a crucial role in the maintenance of cochlear cells. Additional research is required in order to explore the clinical significance of ethosuximide in AHL in humans.

\section{Acknowledgements}

We would like to thank Fengchan Han, Qingzhu Wang and Hui Wang for breeding the NOD/LtJ mice. The present study was supported by grants from National Natural Science Foundation of China (no. 81271085, 81530030 and 81500797), the Natural Science Foundation of Shandong Province (ZR2012HL30, ZR2012HL31 and ZR2014HL050), the Foundation of Taishan Scholar.

\section{References}

1. Gorlin RJ, Toriello HV, and Cohen M (eds): Hereditary Hearing Loss and Its Syndrome. Oxford University Press, 1995.

2. Morton NE: Genetic epidemiology of hearing impairment. Ann NY Acad Sci 630: 16-31, 1991.

3. Mulrow CD, Aguilar C, Endicott JE, Velez R, Tuley MR, Charlip WS and Hill Mulrow CD, Aguilar C, Endicott JE, Velez R, Tuley MR, Charlip WS and Hill JA; JA: Association between hearing impairment and the quality of life of elderly individuals. J Am Geriatr Soc 38: 45-50, 1990.

4. Lin FR, Niparko JK and Ferrucci L: Hearing loss prevalence in the United States. Arch Intern Med 171: 1851-1852, 2001.

5. Appler JM and Goodrich LV: Connecting the ear to the brain: Molecular mechanisms of auditory circuit assembly. Prog Neurobiol 93: 488-508, 2011.

6. Johnson KR and Zheng QY: Ahl2, a second locus affecting agerelated hearing loss in mice. Genomics 80: 461-464, 2002.

7. Noben-Trauth K, Zheng QY and Johnson KR: Association of cadherin 23 with polygenic inheritance and genetic modification of sensorineural hearing loss. Nat Genet 35: 21-23, 2003.

8. Mills JH, Matthews LJ, Lee FS, Dubno JR, Schulte BA and Weber PC: Gender-specific effects of drugs on hearing levels of older persons. Ann NY Acad Sci 884: 381-388, 1999.

9. Youle RJ and Strasser A: The BCL-2 protein family: Opposing activities that mediate cell death. Nat Rev Mol Cell Biol 9: 47-59, 2008.

10. Someya S, Yamasoba T, Weindruch R, Prolla TA and Tanokura M: Caloric restriction suppresses apoptotic cell death in the mammalian cochlea and leads to prevention of presbycusis. Neurobiol Aging 28: 1613-1622, 2007.

11. Someya S, Yamasoba T, Kujoth GC, Pugh TD, Weindruch R, Tanokura $\mathrm{M}$ and Prolla TA: The role of mtDNA mutations in the pathogenesis of age-related hearing loss in mice carrying a mutator DNA polymerase gamma. Neurobiol Aging 29: 1080-1092, 2008.

12. Rogawski MA and Löscher W: The neurobiology of antiepileptic drugs for the treatment of nonepileptic conditions. Nat Med 10: 685-692, 2004.

13. Han F, Yu H, Tian C, Chen HE, Benedict-Alderfer C, Zheng Y, Wang Q, Han X and Zheng QY: A new mouse mutant of the Cdh23 gene with early-onset hearing loss facilitates evaluation of otoprotection drugs. Pharmacogenomics J 12: 30-44, 2012.

14. Kusakawa G, Saito T, Onuki R, Ishiguro K, Kishimoto T and Hisanaga S: Calpain-dependent proteolytic cleavage of the p35 cyclin-dependent kinase 5 activator to p25. J Biol Chem 275: 17166-17172, 2000.

15. Nakagawa $T$ and Yuan J: Cross-talk between two cysteine protease families. Activation of caspase-12 by calpain in apoptosis. J Cell Biol 150: 887-894, 2000.

16. Han F, Yu H, Zheng T, Ma X, Zhao X, Li P, Le L, Su Y and Zheng QY: Otoprotective effects of erythropoietin on Cdh2 $23^{\text {erl/erl }}$ mice. Neuroscience 237: 1-6, 2013.

17. Johnson KR, Erway LC, Cook SA, Willott JF and Zheng QY: A major gene affecting age-related hearing loss in C57BL/6J mice. Hear Res 114: 83-92, 1997.

18. Zheng QY, Johnson KR and Erway LC: Assessment of hearing in 80 inbred strains of mice by ABR threshold analyses. Hear Res 130: 94-107, 1999.

19. Polak M, Eshraghi AA, Nehme O, Ahsan S, Guzman J, Delgado RE, He J, Telischi FF, Balkany TJ and Van De Water TR: Evaluation of hearing and auditory nerve function by combining ABR, DPOAE and eABR tests into a single recording session. J Neurosci Methods 134: 141-149, 2004.

20. Livak KJ and Schmittgen TD: Analysis of relative gene expression data using real-time quantitative PCR and the 2(- $\Delta \Delta C(T))$ method. Methods 25: 402-408, 2001.

21. Perez-Reyes E, Cribbs LL, Daud A, Lacerda AE, Barclay J, Williamson MP, Fox M, Rees M and Lee JH: Molecular characterization of a neuronal low-voltage-activated T-type calcium channel. Nature 391: 896-900, 1998. 
22. Creagh EM, Conroy $\mathrm{H}$ and Martin SJ: Caspase-activation pathways in apoptosis and immunity. Immunol Rev 193: 10-21, 2003.

23. Yin XM: Signal transduction mediated by Bid, a pro-death Bcl-2 family proteins, connects the death receptor and mitochondria apoptosis pathways. Cell Res 10: 161-167, 2000.

24. Azam M, Andrabi SS, Sahr KE, Kamath L, Kuliopulos A and Chishti AH: Disruption of the mouse mu-calpain gene reveals an essential role in platelet function. Mol Cell Biol 21: 2213-2220, 2001.

25. Schuknecht HF and Gacek MR: Cochlear pathology in presbycusis. Ann Otol Rhinol Laryngol 102: 1-16, 1993.

26. Keithley EM, Canto C, Zheng QY, Fischel-Ghodsian N and Johnson KR: Age-related hearing loss and the ahl locus in mice. Hear Res 188: 21-28, 2004.

27. Hudspeth AJ: How hearing happens. Neuron 19: 947-950, 1997.

28. Ehret G: Quantitative analysis of nerve fibre densities in the cochlea of the house mouse (Mus musculus). J Comp Neurol 183. 73-88, 1979.

29. Di Palma F, Pellegrino R and Noben-Trauth K: Genomic structure, alternative splice forms and normal and mutant alleles of cadherin 23 (Cdh23). Gene 281: 31-41, 2001.

30. Di Palma F, Holme RH, Bryda EC, Belyantseva IA, Pellegrino R, Kachar B, Steel KP and Noben-Trauth K: Mutations in Cdh23, encoding a new type of cadherin, cause stereocilia disorganization in waltzer, the mouse model for Usher syndrome type 1D. Nat Genet 27: 103-107, 2001.

31. Siemens J, Lillo C, Dumont RA, Reynolds A, Williams DS, Gillespie PG and Müller U: Cadherin 23 is a component of the tip link in hair-cell stereocilia. Nature 428: 950-955, 2004.

32. Howard $\mathbf{J}$ and Hudspeth AJ: Compliance of the hair bundle associated with gating of mechanoelectrical transduction channels in the bullfrog's saccular hair cell. Neuron 1: 189-199, 1988.

33. Jia S, Dallos P and He DZ: Mechanoelectric transduction of adult inner hair cells. J Neurosci 27: 1006-1014, 2007.

34. Chan DK, Lieberman DM, Musatov S, Goldfein JA, Selesnick SH and Kaplitt MG: Protection against cisplatin-induced ototoxicity by adeno-associated virus-mediated delivery of the X-linked inhibitor of apoptosis protein is not dependent on caspase inhibition. Otol Neurotol 28: 417-425, 2007.

35. Lee S, Briklin O, Hiel H and Fuchs P: Calcium-dependent inactivation of calcium channels in cochlear hair cells of the chicken J Physiol 583: 909-922, 2007.

36. Lopez I, Ishiyama G, Acuna D, Ishiyama A and Baloh RW Immunolocalization of voltage-gated calcium channel alpha1 subunits in the chinchilla cochlea. Cell Tissue Res 313: 177-186, 2003 .
37. Nie L, Zhu J, Gratton MA, Liao A, Mu KJ, Nonner W, Richardson GP and Yamoah EN: Molecular identity and functional properties of a novel T-type $\mathrm{Ca}^{2+}$ channel cloned from the sensory epithelia of the mouse inner ear. J Neurophysiol 100: 2287-2299, 2008.

38. Shen H, Zhang B, Shin JH, Lei D, Du Y, Gao X, Wang Q, Ohlemiller KK, Piccirillo J and Bao J: Prophylactic and therapeutic functions of T-type calcium blockers against noiseinduced hearing loss. Hear Res 226: 52-60, 2007.

39. Becker AJ, Pitsch J, Sochivko D, Opitz T, Staniek M, Chen CC, Campbell KP, Schoch S, Yaari Y and Beck H: Transcriptional upregulation of Cav3.2 mediates epileptogenesis in the pilocarpine model of epilepsy. J Neurosci 28: 13341-13353, 2008.

40. Deshpande LS, Limbrick DD Jr, Sombati S and DeLorenzo RJ: Activation of a novel injury-induced calcium-permeable channel that plays a key role in causing extended neuronal depolarization and initiating neuronal death in excitotoxic neuronal injury. J Pharmacol Exp Ther 322: 443-452, 2007.

41. Matsunami M, Kirishi S, Okui T and Kawabata A: Hydrogen sulfide-induced colonic mucosal cytoprotection involves T-type calcium channel-dependent neuronal excitation in rats. J Physiol Pharmacol 63: 61-68, 2012.

42. Lei D, Gao X, Perez P, Ohlemiller KK, Chen CC, Campbell KP, Hood AY and Bao J: Anti-epileptic drugs delay age-related loss of spiral ganglion neurons via T-type calcium channel. Hear Res 278: 106-112, 2011.

43. Gao M, Wong SY, Lau PM and Kong SK: Ferutinin induces in vitro eryptosis/erythroptosis in human erythrocytes through membrane permeabilization and calcium influx. Chem Res Toxicol 26: 1218-1228, 2013.

44. Ding D, Stracher A and Salvi RJ: Leupeptin protects cochlear and vestibular hair cells from gentamicin ototoxicity. Hear Res 164: 115-126, 2002.

45. Coulter DA, Sombati S and DeLorenzo RJ: Electrophysiology of glutamate neurotoxicity in vitro: Induction of a calciumdependent extended neuronal depolarization. J Neurophysiol 68: 362-373, 1992.

46. Limbrick DD Jr, Pal S and DeLorenzo RJ: Hippocampal neurons exhibit both persistent $\mathrm{Ca}^{2+}$ influx and impairment of $\mathrm{Ca}^{2+}$ sequestration/extrusion mechanisms following excitotoxic glutamate exposure. Brain Res 894: 56-67, 2001. 